PROCEEDINGS OF THE

AMERICAN MATHEMATICAL SOCIETY

Volume 125, Number 5, May 1997, Pages 1259-1262

S 0002-9939(97)03801-X

\title{
FINITE LINEAR GROUPS AND THEOREMS OF MINKOWSKI AND SCHUR
}

\author{
WALTER FEIT
}

(Communicated by Ronald M. Solomon)

\begin{abstract}
Let $G$ be a finite group with a faithful rational valued character of degree $n$. A theorem of I. Schur gives a bound for the order of $G$ in terms of $n$, generalizing an earlier result of $\mathrm{H}$. Minkowski who showed that the same bound holds if $G \subseteq G L(n, \mathbf{Q})$. This note contains strengthened versions of these results which in particular show that a 2 -subgroup of $G L(n, \mathbf{Q})$ of maximum possible order contains a reflection.
\end{abstract}

\section{§1. Statements of Results}

If $\ell$ is a natural number, let $\mathbf{Q}(2 \ell)$ denote the cyclotomic field which contains exactly $2 \ell$ roots of 1 , thus $\mathbf{Q}(2)=\mathbf{Q}$. For natural numbers $\ell$ and $m$, let $M(m, \ell)$ denote the group of all monomial matrices whose non-zero entries are $\ell$ th roots of 1. Then $|M(m, \ell)|=\ell^{m}(m !)$.

Let $p$ be a prime. For a natural number $u$ let $u_{p}$ denote the $p$-part of $u$. If $\ell_{p}=p^{k}$, let $P\left(m, p^{k}\right)$ be a Sylow $p$-group of $M(m, \ell)$. As

$$
|M(m, \ell)|_{p}=p^{k m}(m !)_{p}=\left|M\left(m, p^{k}\right)\right|_{p},
$$

it may be assumed that $P\left(m, p^{k}\right) \subseteq M\left(m, p^{k}\right)$. Furthermore, $\left|P\left(m, p^{k}\right)\right|=p^{N_{p}\left(m, p^{k}\right)}$, with

$$
N_{p}\left(m, p^{k}\right)=m k+\sum_{i \geq 1}\left[m / p^{i}\right] .
$$

Theorem A. Let $p$ be a prime. If $p=2$ let $\ell=2$, otherwise let $\ell=2 p$. Let $P$ be a finite p-group contained in $G L(m, \mathbf{Q}(\ell))$. Then $P$ is conjugate in $G L(m, \mathbf{Q}(\ell))$ to a subgroup of $M(m, p)$.

In case $p=2$ Theorem A becomes

Theorem B. Let $P$ be a finite 2-group contained in $G L(n, \mathbf{Q})$. Then $P$ is conjugate in $G L(n, \mathbf{Q})$ to a subgroup of $M(n, 2)$.

An immediate consequence of Theorem B is

Theorem C. If $G$ is a finite group contained in $G L(n, \mathbf{Q})$ with $|G|_{2}=|M(n, 2)|_{2}$, then $G$ contains a reflection.

Received by the editors November 1, 1995.

1991 Mathematics Subject Classification. Primary 20C15; Secondary $20 \mathrm{H} 20$.

(c)1997 American Mathematical Society 
For general $p$, we will prove

Theorem D. If $P$ is a finite p-group contained in $G L(n, \mathbf{Q})$, then $P$ is isomorphic to a subgroup of $P([n /(p-1)], p)$.

If $p$ is odd, Theorem D is known, see [B]. (I am indebted to J.-P. Serre for drawing my attention to this reference). A proof is included here as the methods of this paper are quite different from those in [B]. If $p=2$, Theorem $\mathrm{D}$ is a weak form of Theorem B, however Theorem B does not appear to be in the literature.

We will also prove

Theorem E. Let $P$ be a finite p-group which has a faithful irreducible Q-valued character of degree $n$ and let $m=[n /(p-1)]$. Then $|P| \leq|P(m, p)|=p^{N_{p}(m, p)}$.

Theorem E and (1.1) imply the following result of Schur, [S, vol.1, p.129].

Theorem F. If $G$ is a finite group which has a faithful $\mathbf{Q}$-valued character of degree $n$, then $|G|$ divides $\prod_{p} p^{M_{p}(n)}$, with

$$
M_{p}(n)=N_{p}([n /(p-1)], p)=\sum_{i \geq 0}\left[n /\left(p^{i}(p-1)\right)\right] .
$$

Theorem $\mathrm{F}$ generalizes an earlier result of Minkowski, who reached the same conclusion under the assumption that $G \subseteq G L(n, \mathbf{Q})$.

Theorem F occurs in one of Schur's earliest papers, it is the $6^{\text {th }}$ in his collected works. The methods of this note are based on ideas that were introduced by Schur in later work. The starting point is Theorem 2.1 below. This is closely related to L. Solomon's thesis, which involves a natural continuation of ideas that go back to Schur and R. Brauer.

\section{§2. Proofs}

Let $\theta$ be a character of $G$ of degree $n$. Suppose that $\theta=\lambda^{G}$ for a linear character $\lambda$ of some subgroup of $G$. If $\lambda^{\ell}=1$ for some $\ell$, then a representation $f$ which affords $\theta$ can be chosen so that $f(G) \subseteq M(n, \ell)$. This observation will be used frequently below.

The following result is a special case of $[\mathrm{F},(14.3)]$.

Theorem 2.1. Let $p$ be a prime and let $P$ be a p-group. Let $K$ be a number field and let $\chi$ be a faithful irreducible nonlinear character of $P$ with values in $K$. Assume that one of the following holds:

(i) $p$ is odd,

(ii) $p=2$ and $P$ does not contain a cyclic subgroup of index 2 .

Then there exists $P_{0} \triangleleft P$ with $\left[P: P_{0}\right]=p$ and an irreducible character $\zeta$ of $P_{0}$ with values in $K$ such that $\zeta^{P}=\chi$.

As a consequence, we get

Theorem 2.2. Let $p$ be a prime and let $P$ be a p-group. Let $K$ be a number field and let $\chi$ be an irreducible character of $P$ with values in $K$. Let $S$ be a subgroup of $P$ of minimum order such that $\chi=\eta^{P}$ for an irreducible character $\eta$ of $S$ with values in $K$. Then one of the following holds:

(i) $\eta$ is linear and $\chi$ is afforded by a $K[P]$ module (i.e. the Schur index of $\chi$ over $K$ is 1$)$.

(ii) $p=2$ and $\eta(1)=2$. Furthermore, if $S_{0}$ is the kernel of $\eta$, then $\bar{S}=S / S_{0}$ has a cyclic subgroup $C$ of index 2. 
Proof. If $\eta$ is linear, (i) holds. Suppose that $\eta$ is not linear. By Theorem 2.1 the minimality of $|S|$ implies that $p=2$ and $\bar{S}$ contains a cyclic subgroup $C$ with $[\bar{S}: C]=2$. Hence, $\eta(1)=2$ as required.

Theorem 2.3. Let $T$ be a nonabelian 2-group which has a cyclic subgroup $C=\langle x\rangle$ of index 2. Let $|T|=2^{k+1}$. Then $T=\langle x, y\rangle$ with $y^{-1} x y=x^{m}$ and $m^{2} \equiv 1$ $\left(\bmod 2^{k}\right), m \neq \equiv 1\left(\bmod 2^{k}\right)$. Let $\eta$ be a faithful irreducible character of $T$.

(i) Then $\eta(1)=2$ and $[\mathbf{Q}(\eta): \mathbf{Q}]=2^{k-2}$.

(ii) Either $T$ has a noncyclic subgroup $H$ of order 4 or $T$ is a generalized quaternion group and contains a quaternion subgroup $H$ of order 8.

(iii) Suppose that $T$ contains a noncyclic subgroup $H$ of order 4 . Then there is a Q-valued linear character $\lambda$ of $H$ with $\lambda^{T}=\sum \eta^{\sigma}$, where $\sigma$ ranges over the Galois group of $\mathbf{Q}(\eta) / \mathbf{Q}$.

(iv) Suppose that $T$ is a generalized quaternion group. Let $H$ be a quaternion subgroup of $T$ of order 8 . Let $\rho$ be the unique irreducible character of $H$ of degree 2. Then $\eta$ is real valued but is not afforded by an $\mathbf{R}[T]$ module (i.e. the Schur index over $\mathbf{R}$ is 2). Furthermore $\sum \eta^{\sigma}=\rho^{T}$ and $2 \sum \eta^{\sigma}=\lambda^{T}$, where $\lambda$ is the nonprincipal character of the center of $H$.

Proof. (i) $\eta_{C}=\mu+\mu^{\prime}$, where $\mu$ and $\mu^{\prime}$ are faithful linear characters of $C$. As $\mu^{\prime}=\mu^{m},[\mathbf{Q}(\mu): \mathbf{Q}(\eta)]=2$. Hence $[\mathbf{Q}(\eta): \mathbf{Q}]=2^{k-2}$ as $\mathbf{Q}(\mu)=\mathbf{Q}(|C|)=\mathbf{Q}\left(2^{k}\right)$.

(ii) It is well known that a noncyclic 2-group which contains only one involution is a generalized quaternion group and contains a quaternion subgroup of order 8 . If $T$ contains an involution outside the center, then it clearly contains a noncyclic group of order 4.

(iii) As $H$ is not cyclic, $\eta_{H}=\lambda+\lambda^{\prime}$, with $\lambda \neq \lambda^{\prime}$ and $\lambda$ necessarily Q-valued. Since $\eta$ has $2^{k-2}$ algebraic conjugates, the result follows from Frobenius reciprocity as $\lambda^{T}(1)=2^{k-1}$.

(iv) $\eta_{H}^{\sigma}=\rho$ for every algebraic conjugate $\eta^{\sigma}$ of $\eta$. As $\eta$ has $2^{k-2}$ algebraic conjugates, $\rho^{T}=\sum \eta^{\sigma}$ by Frobenius reciprocity. Since $m=-1, \rho \in \mathbf{R}$. As is well known, $\rho$ is not afforded by an $\mathbf{R}[H]$ module. Therefore, $\rho$ has Schur index 2 over $\mathbf{R}$ as it is $\mathbf{R}$-valued, in fact $\mathbf{Q}$-valued. The remaining statement is a consequence of the fact that $2 \rho=\lambda^{H}$.

Theorem 2.4. Let $p$ be a prime. If $p=2$ let $\ell=2$, otherwise let $\ell=2 p$. Let $K=$ $\mathbf{Q}(\ell)$ and let $P$ be a p-group. Let $f$ be a faithful irreducible $K[P]$-representation. Then $f$ can be chosen to be an isomorphism of $P$ into $M(n, p)$.

Proof. It may be assumed that $|P|>1$. Let $\zeta$ be the character afforded by $f$. Then $\zeta=m_{K}(\chi) \sum \chi^{\sigma}$, where $\chi$ is a faithful irreducible character of $P, \sigma$ ranges over the Galois group of $K(\chi) / K$ and $m_{K}(\chi)$ is the Schur index of $\chi$ over $K$.

Suppose that Theorem 2.2 (i) holds for $\chi$. Let $S_{1}$ be the subgroup of order $p$ of $S$ and let $\eta_{S_{1}}=\lambda$. Then $\lambda^{P}=\zeta$ and the result follows.

Suppose that Theorem 2.2 (ii) holds. Then $p=2$ and $K=\mathbf{Q}$. Let $T=S$ in Theorem 2.2, and apply Theorem 2.3 .

If $|H|=4$, Theorem 2.3 (iii) implies that $m_{K}(\chi)=m_{K}(\eta)=1$, and so $\zeta=\lambda^{T}$ for a $\mathbf{Q}$-valued linear character $\lambda$ of $H$.

If $H$ is a quaternion group, then $m_{\mathbf{Q}}(\chi)=2$, and so by Theorem 2.3 (iv), $\zeta=\lambda^{P}$ for a $\mathbf{Q}$-valued linear character $\lambda$ of the center of $H$. 
Observe that if $\Sigma n_{i}=n$, then for all $\ell$

$$
M\left(n_{1}, \ell\right) \times M\left(n_{2}, \ell\right) \times \ldots \subseteq M(n, \ell) .
$$

Hence also

$$
\prod\left|M\left(n_{i}, \ell\right)\right||| M(n, \ell) \mid
$$

Note also that since a $p$-group $P \neq\langle 1\rangle$ has a nontrivial center, $\mathbf{Q}(2 \ell) \subseteq \mathbf{Q}(\chi)$ for any nonprincipal irreducible character $\chi$ of $P$.

Proof of Theorem $A$. Let $\zeta(x)=\operatorname{trace}(x)$ for $x \in P$; then $\zeta$ is a character of the group $P$, which is afforded by a $\mathbf{Q}(\ell)[P]$ module. Thus $\zeta=t 1_{P}+\sum \zeta_{i}$, where each $\zeta_{i}$ is afforded by a nontrivial irreducible $\mathbf{Q}(\ell)$-module. The result follows from Theorem 2.4 and (2.5).

As remarked in the introduction, Theorems B and C follow from Theorem A.

Proof of Theorem D. By Theorem B, it may be assumed that $p$ is odd. Let $\theta(x)=$ $\operatorname{trace}(x)$ for $x \in P$. Then $\theta=t 1_{P}+\theta^{\prime}$ with $\theta^{\prime}=\sum \theta_{i}$, where each $\theta_{i}$ is afforded by a nontrivial irreducible $\mathbf{Q}[P]$ module. By Theorem 2.2 each $\theta_{i}=\sum \chi_{i}^{\sigma}$, where $\chi_{i}$ is a nontrivial irreducible character of $P, \mathbf{Q}\left(\chi_{i}\right)=\mathbf{Q}\left(2 p^{s_{i}}\right)$ for some $s_{i}>0$ and $\sigma$ ranges over the Galois group of $\mathbf{Q}\left(\chi_{i}\right) / \mathbf{Q}$. Therefore $\theta_{i}=\sum \zeta_{i}^{\tau}$, where $\mathbf{Q}\left(\zeta_{i}\right)=\mathbf{Q}(2 p)$ and $\tau$ ranges over the Galois group of $\mathbf{Q}\left(\zeta_{i}\right) / \mathbf{Q}$. Thus $\zeta_{i}=\sum \chi_{i}^{\sigma}$, where $\sigma$ ranges over the Galois group of $\mathbf{Q}\left(\chi_{i}\right) / \mathbf{Q}\left(\zeta_{i}\right)$. If $f_{i}$ is a $\mathbf{Q}\left(\zeta_{i}\right)[P]$-representation of $P$ afforded by $\zeta_{i}$, then by Theorem $2.2 f_{i}=f$ can be chosen so that $f_{i}(P) \subseteq M\left(\zeta_{i}(1), p\right)$. Hence by (2.5), $P$ is isomorphic to a subgroup of $P\left(\theta^{\prime}(1) /(p-1), p\right)$. The result follows as

$$
\theta^{\prime}(1) /(p-1)=\left[\theta^{\prime}(1) /(p-1)\right] \leq[n /(p-1)] .
$$

As Theorem E and (1.1) imply Theorem F, it remains only to prove Theorem E.

Proof of Theorem E. Let $\theta$ be a $\mathbf{Q}$-valued character of $P$. Then $\theta=\sum \zeta_{i}$ where for each $i, \zeta_{i}=\sum \chi_{i}^{\sigma}$, with $\chi_{i}$ an irreducible character of $P$ and $\sigma$ ranging over the Galois group of $\mathbf{Q}\left(\chi_{i}\right) / \mathbf{Q}$. In view of (2.5) and (2.6) it suffices to prove the result in case $\theta=\zeta=\sum \chi^{\sigma}$, with $\chi$ a faithful irreducible character of $P$. If $\chi$ has Schur index $m_{\mathbf{Q}}(\chi)=1$ over $\mathbf{Q}$ then a representation of $P$ which affords $\chi$ maps $P$ isomorphically into $G L(n, \mathbf{Q})$ and (i) holds by Theorem D.

Suppose that $m_{\mathbf{Q}}(\chi)>1$. By Theorem 2.2, $p=2$. By Theorem 2.3, $P$ contains a quaternion group $H$ of order 8 and $\theta=\rho^{P}$, where $\rho$ is the unique irreducible character of $H$ of degree 2 . This implies that $|P| \leq 8^{t}(t !)$, where $n=2 t$. Thus $|P|=2^{k}$, with

$$
k \leq 3 t+\sum_{i \geq 1}\left[t / 2^{i}\right]=n+n / 2+\sum_{i \geq 2}\left[n / 2^{i}\right]=N_{2}(n, 2)
$$

as required.

\section{REFERENCES}

[B] N. Bourbaki, Groupes et algèbres de Lie, Chapitres 3, §7, Exercises 5-8. MR 58:28083a

[F] W. Feit, Characters of finite groups, Benjamin, NY, Amsterdam (1967). MR 36:2715

[S] I. Schur, Collected Works, Springer-Verlag, Berlin, Heidelberg, N.Y. MR 57:2858a

Department of Mathematics, Yale University, P.O. Box 208283, New Haven, ConNECTICUT 06520-8283

E-mail address: feit@math.yale.edu 